\title{
Incidência e mortalidade por AIDS em crianças e adolescentes: desafios na região sul do Brasil
}

\author{
Incidence and mortality of children and teenagers with AIDS: \\ challenges in the southern region of Brazil
}

Márcio Cristiano de Melo ${ }^{1}$

Rosemeire de Olanda Ferraz ${ }^{1}$

Juliana Luporini do Nascimento ${ }^{1}$

Maria Rita Donalisio ${ }^{1}$

${ }^{1}$ Departamento de Saúde Coletiva, Faculdade de Ciências Médicas, Universidade Estadual de Campinas. R. Tessália Vieira de Camargo 126, Cidade Universitária. 13083-887 Campinas SP Brasil. enf.marciomelo@gmail.com
Abstract The objective of this study is to describe the time trend of coefficients of incidence and mortality associated with AIDS in children and adolescents. An ecological time trend study was conducted among individuals under 14 living in the regional context of high incidence of AIDS - Porto Alegre and the State of Rio Grande do Sul - from 1996 to 2012. For this purpose, a segmented regression model was adjusted for age categories and place of residence. AIDS morbidity and mortality indicators in Porto Alegre are still higher than those registered in the State, the southern region and other areas of the country. In Porto Alegre, 59.1\% of cases (371/628) occurred in children under four years of age, the incidence showed peaks in the period and the mortality dropped from 9 to 2 per 100,000. A significant decreasing in annual percentage change in mortality was observed especially in children under 1 year of age in Porto Alegre. This drop may be related to prevention and treatment of pregnant women. The challenge is to identify possible fragilities of the STD/AIDS Program and its vulnerable points in order to make specific interventions and investments with a greater impact potential.

Key words HIV/AIDS, Child, Adolescent, Incidence, Mortality rate
Resumo O objetivo deste estudo é descrever a tendência temporal dos coeficientes de incidência e mortalidade associados à AIDS em crianças e adolescentes. Foi desenvolvido um estudo ecológico de tendência temporal dos indicadores em menores de 14 anos residentes em contexto regional de alta incidência de AIDS, Porto Alegre e estado do Rio Grande do Sul, de 1996 a 2012. Para tanto, se ajustou modelo de regressão segmentada para categorias de idade e local de residência. Os indicadores de morbimortalidade da AIDS em Porto Alegre ainda superam os registrados no estado, na região Sul e em outros do país. Em Porto Alegre, $59,1 \%$ dos casos (371/628) ocorreram em menores de quatro anos, a incidência apresentou picos no período e a mortalidade caiu de 9 para 2 por 100 mil. Registrou-se variação anual percentual decrescente significativa na mortalidade principalmente de menores de 1 ano em Porto Alegre. Esta queda pode estar relacionada com a prevenção $e$ o tratamento de gestantes. Resta identificar possíveis fragilidades do Programa DST/AIDS e seus pontos vulneráveis para intervenções específicas e investimentos com maior potencial de impacto.

Palavras-chave HIV/AIDS, Criança, Adolescente, Coeficiente de incidência, Coeficiente de mortalidade 


\section{Introdução}

O vírus da imunodeficiência humana (HIV) pertence à classe dos retrovírus e é o agente etiológico da Síndrome da Imunodeficiência Adquirida (AIDS) ${ }^{1}$. A transmissão pode ocorrer pelas vias sexual, sanguínea ou parenteral, quando há o contato e/ou a troca de sangue ou secreção orgânica contendo o vírus ou por células parasitadas ${ }^{2}$.

Nas últimas décadas, o acometimento pela infecção HIV/AIDS em mulheres na idade reprodutiva (de 15 a 49 anos) gerou um aumento no número de crianças de 0 a 14 anos infectadas pelo HIV, em que a transmissão vertical foi responsável por $84 \%$ dos casos ${ }^{3}$.

A epidemia de HIV/AIDS pode impactar a qualidade de vida de crianças e adolescentes acometidas pela infecção, com a possibilidade destes passarem por perdas sucessivas, declínio da saúde e mudanças no convívio com pais e parentes ${ }^{4}$. Crianças e adolescentes vivendo com HIV/AIDS merecem atenção especial já que apresentam singularidades em suas vivências relacionadas à perda de familiares, à discriminação e preconceito e à possibilidade eminente de adoecimento e morte. Estas questões interferem diretamente na sua qualidade de vida $\mathrm{e}$ conferem um lugar social distinto das outras crianças e adolescentes da mesma faixa etária ${ }^{5}$.

A partir de 1996, o Programa Nacional de DST-AIDS disponibilizou a terapia antirretroviral de alto impacto (HAART) e ampliou as ações profiláticas, particularmente à gestante infectada e ao recém-nascido exposto, reduzindo a morbimortalidade relacionada à infecção pelo HIV/ AIDS em crianças, mesmo durante a expansão da epidemia entre mulheres ${ }^{6-8}$.

Nos últimos anos houve uma diminuição dos coeficientes de transmissão vertical do HIV no Brasil, embora a taxa de deteç̧ão em gestantes tenha aumentado. Tem sido observada uma nítida mudança no cenário da infecção pelo HIV em crianças, além do aumento da sobrevida em função do acesso aos medicamentos e cuidados. Tal realidade permite que crianças portadoras do HIV cheguem à adolescência e, finalmente, à idade adulta, em novo contexto epidemiológico, diferente do que se visualizava, por exemplo, no final da década de 1980 e nos anos 90, período em que se intensificou o processo de feminização, juvenialização e pauperização da epidemia $a^{5,9}$.

Estudo realizado na cidade do Rio de Janeiro registrou rápido aumento da incidência da AIDS entre mulheres e diminuição da razão de casos homem/mulher, de 4,7 , no final dos anos 80 , para 0,5 , no período de 2005 a $2009^{10}$.
A região Sul do Brasil destaca-se tanto pela alta taxa de detecção da doença, 41,3/100.000 habitantes em 2013, como por ser a região onde se encontram as 14 cidades com mais de 50 mil habitantes que possuem as maiores taxas no país. Ao se utilizar o índice composto (detecção, mortalidade e primeiro CD4) para o ranking dos estados, o Rio Grande do Sul lidera a classificação de risco no Brasil ${ }^{11}$.

Em 2013, este estado apresentou taxa de deteç̧ão em menores de cinco anos de 9,1/100.000 habitantes, acima da média nacional, de 3,4/100.000 habitantes, registrando as maiores taxas desde $2006^{7}$. No Brasil, a taxa de detecção de gestantes com HIV por 1.000 nascidos vivos aumentou na última década, atingindo 2,5 casos para cada mil nascidos vivos, em 2013. Na região Sul este indicador foi 2,3 vezes maior que a média do país ${ }^{11}$.

Embora em cidades com mais de 500 mil habitantes se observe a desaceleração da incidência da AIDS, esta tendência não aparece na região Sul, com indicadores de risco de infecção e de morte que superam os da região Sudeste ${ }^{7}$.

Em função da alta incidência de AIDS na região Sul do país, principalmente na cidade de Porto Alegre, o objetivo deste trabalho consiste em analisar a tendência temporal dos coeficientes de incidência e mortalidade associados à AIDS em crianças e adolescentes com até 14 anos residentes em Porto Alegre.

\section{Método}

Estudo ecológico da tendência temporal dos coeficientes de incidência de AIDS por 100.000 crianças menores de 14 anos residentes em Porto Alegre, no estado do Rio Grande do Sul, nos anos de 1996 a 2012. Foi analisada a totalidade dos casos notificados no Sistema de Informações de Agravos de Notificação (SINAN) e dos óbitos registrados no Sistema de Informação sobre Mortalidade (SIM). Por se tratar de indicadores específicos por faixa etária não foi necessária a padronização dos coeficientes.

Porto Alegre é a capital do estado do RS, com 1.467.823 habitantes ${ }^{12}$, à beira do lago Guaíba e com densidade populacional acima de $2000 \mathrm{hab} /$ $\mathrm{km}^{2}$. A alta incidência de AIDS no estado e na capital, comparada com outras áreas do país, justificou a escolha da região de estudo.

O início do período de estudo (1996) coincide com a disponibilização da HAART e a universalização de medidas profiláticas à gestante $\mathrm{e}$ 
ao recém-nascido pelo Programa DST/AIDS no país, além da disponibilidade desses dados no Departamento de Informática do Sistema Único de Saúde (DATASUS). O término do período (2012) foi delimitado para se obter estatísticas consolidadas nos bancos de dados oficiais.

Foram analisadas as seguintes variáveis constantes na ficha de notificação epidemiológica do SINAN no DATASUS: sexo, raça/cor (branca, preta, amarela, parda, indígena, ignorada) e faixa etária detalhada (menor 1 ano, 1 a 4 anos, 5 a 9 anos, 10 a 14 anos). Dados de mortalidade foram obtidos do SIM, referentes aos diagnósticos de causa básica, obtidos pela Classificação Internacional de Doenças 10a edição - CID-10: de B-20 a B-24.

Como critério de inclusão no estudo, utilizou-se a faixa etária de menores de 14 anos e o local de moradia, (Porto Alegre, estado RS e região Sul) na seleção dos dados disponibilizados nos bancos referentes ao período de 1996 a 2012.

Os coeficientes de incidência e mortalidade foram calculados utilizando-se os casos de AIDS notificados e os óbitos decorrentes da mesma causa como numeradores, respectivamente, e as populações das faixas etárias dos censos demográficos, como denominadores para cada 100.000 habitantes.

Seguem as fórmulas para o cálculo dos coeficientes de mortalidade específicos e dos coeficientes de incidência:

\begin{tabular}{|c|c|}
\hline $\begin{array}{c}\text { Coeficiente } \\
\text { de Incidência }\end{array}$ & $\begin{array}{c}\mathrm{n}^{\mathrm{o}} \text { casos de AIDS } \\
\text { na faixa etária de estudo } \\
\text { população da faixa etária }\end{array}$ \\
\hline $\begin{array}{l}\text { Coeficiente de } \\
\text { Mortalidade }= \\
\text { Específico }\end{array}$ & $\begin{array}{c}\text { nº de óbitos por AIDS } \\
\text { (CID-10: B-20 a B-24) } \\
\text { na faixa etária de estudo } \\
\text { população da faixa etária }\end{array}$ \\
\hline
\end{tabular}

As tendências dos coeficientes de mortalidade por AIDS foram analisadas por meio de regressão segmentada (joinpoint regression) e pela técnica de suavização por médias móveis de três pontos. Para o ajuste das regressões foram consideradas as variáveis dependentes faixas etárias e região de estudo, e como variável independente os anos.

Na técnica de suavização por médias móveis com três pontos, cada observação na série suavizada representa a média de três pontos adjacentes da série original.

A variação anual percentual (Annual Percentual Change, APC) é calculada por meio de uma regressão segmentada que verifica os pontos de mudança (ou ponto de inflexão), apontando variações significativas no incremento da reta. São ajustados sucessivos segmentos de reta conectados entre si por esses pontos de mudança. O número de pontos necessários para o ajuste de cada segmento de regressão e o modelo mais adequado foram selecionados automaticamente pela configuração padrão do programa (sem a transformação logarítmica da variável resposta). Alterou-se apenas a pressuposição de homocedasticidade e a autocorrelação dos resíduos dos modelos mais adequadas para os ajustes, as quais foram verificadas utilizando-se o software estatístico Minitab versão 13.20. Também foram calculados intervalos de confiança de 95\% para o APC. Quando a suposição de homoscedasticidade não é satisfeita, o modelo de regressão segmentada é ajustado por uma regressão ponderada, na qual o peso é o inverso da variabilidade das observações nas variáveis dependentes ${ }^{13,14}$.

Os dados foram apresentados em forma de tabelas e gráficos com as tendências de coeficiente de incidência e de mortalidade de AIDS, com suavização por médias móveis e regressão segmentada (joinpoint regression), variações percentuais anuais com os respectivos intervalos de confiança de $95 \%$ e teste qui-quadrado com correção de Yates (considerando 5\% de significância para os testes estatísticos). Para tanto, foi utilizado o Software Excel 2010 para Windows e o programa de tabulação de dados TabNet do Ministério da Saúde. Também foi utilizado o Joinpoint Regression Program versão 4.1.1.5, fornecido pelo Instituto Nacional de Câncer dos Estados Unidos (Nacional Cancer Institute) $^{15,16}$.

Foram comparados os indicadores de Porto Alegre com os do restante do estado do Rio Grande do Sul (sem os dados da capital) e os da região Sul com a finalidade de observar a progressão da doença e o desfecho óbito no contexto regional de alta incidência de AIDS no Brasil. Para a comparação entre as raças referidas foram excluídos os “amarelos" e os "indígenas" devido ao número pequeno ou nulo de registro de casos ou óbitos.

O projeto foi aprovado pelo Comitê de Ética de Pesquisas em Seres Humanos- FCM/Unicamp.

\section{Resultados}

Em Porto Alegre foram notificados 648 casos de AIDS em menores de 14 anos, sendo 386 (59,6\%) com menos de quatro anos. Ao se comparar com os dados do estado, a capital concentrou 41,7\% dos 1.559 casos da faixa etária, no período de 
1996 a 2012, sendo 936 (60\%) em menores de quatro anos.

Foram registrados 186 óbitos na capital, correspondendo a 35,3\% dos 527 registrados no Rio Grande do Sul. Em crianças com até quatro anos foram $151(81,2 \%)$ óbitos na capital do estado, sendo 101 masculinos (54,3\%). As 405 mortes em crianças de zero a quatro anos no estado corresponderam a $77 \%$ do total por esta causa, sendo $270(51,2 \%)$ femininos.

A Tabela 1 mostra a distribuição dos casos e dos óbitos por AIDS em Porto Alegre e no restante do estado. Observa-se maior concentração de casos em menores de quatro anos (59,6\%). Existe semelhança na distribuição proporcional dos casos e dos óbitos entre Porto Alegre e o restante do estado em relação ao sexo e à faixa etária $(\mathrm{p}>0,05)$. Porém, as proporções de casos e óbitos entre negros e pardos na capital (29,8\% dos casos, $28 \%$ dos óbitos) são maiores que no restante do estado $(11,9 \%$ e $10,1 \%$, respectivamente) $(\mathrm{p}<0,0001)$.

Os modelos de regressão segmentada foram ajustados para todas as categorias de idades e de regiões de estudo. Analisando-se os resíduos dessas regressões, constatou-se que os mesmos eram heterocedásticos e não autocorrelacionados, sendo incorporado aos modelos. Assim, os modelos de regressão ponderada foram ajustados com pe- sos como sendo o inverso da variância dos pontos das respectivas variáveis dependentes (Tabela 2).

Conforme a Tabela 2, houve queda significativa nos coeficientes de mortalidade por AIDS de crianças menores de 1 ano, no período de 1996 a 2003, com maior variação anual percentual, de $-11,14 \%$ ao ano (IC: $-12,11 \%$ a $-10,16 \%$ ), enquanto que no período seguinte a redução anual foi menor, de $-1,58 \%$ ao ano (IC: $-2,49$ a -0,66). $\mathrm{Na}$ faixa etária de 1 a 4 anos houve redução de $-2,07 \%$, no período de 1998 a 2006 (IC: $-3,45$ a $-0,69)$, enquanto que na de 10 a 14 anos ocorreu queda de $-1,23 \%$, no período de 2007 a 2011 (IC: $-3,28$ a 0,83$)$.

A região Sul manteve coeficientes de mortalidade praticamente estáveis ao longo do período de 1996 a 2012, com uma discreta redução de APC de $-0,03 \%$ no período de 2004 a 2012 (IC: $-0,05$ a -0,01). O Estado do Rio Grande do Sul apresentou ligeira tendência decrescente no mesmo período, enquanto que a capital Porto Alegre apresentou dois pontos de mudança no período, com maior decréscimo nos anos de 1998 a 2004, APC de -1,20\% (IC: $-2,16 \%$ a -0,23\%) (Tabela 2).

Os indicadores de incidência e mortalidades de Porto Alegre são comparados com os de todo o estado do Rio Grande do Sul e os da região Sul. Nota-se um aumento na incidência por 100.000

Tabela 1. Percentual de casos e óbitos de AIDS em menores de 14 anos e variáveis sócio demográficas em Porto Alegre (POA) e restante do estado Rio Grande do Sul (RS), 1996 a 2012.

\begin{tabular}{|c|c|c|c|c|c|c|c|c|c|c|}
\hline & \multicolumn{5}{|c|}{ Casos } & \multicolumn{5}{|c|}{ Óbitos } \\
\hline & \multicolumn{2}{|c|}{ RS } & \multicolumn{2}{|c|}{ POA } & \multirow[b]{2}{*}{ p valor ${ }^{*}$} & \multicolumn{2}{|c|}{ RS } & \multicolumn{2}{|c|}{ POA } & \multirow[b]{2}{*}{ p valor ${ }^{*}$} \\
\hline & $\mathbf{n}$ & $\%$ & $\mathbf{n}$ & $\%$ & & $\mathbf{n}$ & $\%$ & $\mathbf{n}$ & $\%$ & \\
\hline Sexo & & & & & 0,612 & & & & & 0,194 \\
\hline Masculino & 761 & 48,8 & 324 & 50,0 & & 257 & 48,8 & 101 & 54,3 & \\
\hline Feminino & 798 & 51,2 & 324 & 50,0 & & 270 & 51,2 & 85 & 45,7 & \\
\hline Faixa Etária & & & & & 0,988 & & & & & 0,868 \\
\hline$<1$ ano & 400 & 25,7 & 162 & 25,0 & & 248 & 47,1 & 93 & 50,0 & \\
\hline 1 a 4 anos & 536 & 34,2 & 224 & 34,6 & & 166 & 31,5 & 58 & 31,2 & \\
\hline 5 a 9 anos & 398 & 25,5 & 166 & 25,6 & & 53 & 10,1 & 16 & 8,6 & \\
\hline 10 a 14 anos & 225 & 14,4 & 96 & 14,8 & & 60 & 11,4 & 19 & 10,2 & \\
\hline Raça/Cor & & & & & $<0,001^{* *}$ & & & & & $0,002^{* *}$ \\
\hline Branca & 843 & 54,1 & 297 & 45,8 & & 349 & 66,2 & 98 & 52,7 & \\
\hline Preta & 186 & 11,9 & 110 & 17,5 & & 56 & 10,6 & 34 & 18,3 & \\
\hline Amarela & 1 & 0,1 & - & - & & 1 & 0,2 & - & - & \\
\hline Parda & 157 & 10,1 & 83 & 12,8 & & 44 & 8,3 & 18 & 9,7 & \\
\hline Indígena & 4 & 0,3 & 2 & 0,3 & & - & - & - & - & \\
\hline Ignorado & 367 & 23,5 & 156 & 24,1 & & 76 & 14,4 & 36 & 19,4 & \\
\hline
\end{tabular}

Fonte: MS/SVS/DASIS - Sistema de Informações sobre Mortalidade - SIM. MS/SVS/DATASUS - Sistema de Informação de Agravos de Notificação - SINAN. n: número absoluto de casos/óbitos. " p-valor obtido do Teste Qui-Quadrado com correção de Yates. ${ }^{* *}$ Para o cálculo foram excluídas as raças amarela e indígena. 
habitantes em 2001, 2002, 2007 e 2011, em Porto Alegre, quando comparados com o estado e a região, maior no período de 2000 a 2002, conforme mostra a série suavizada (Figura 1A). Destacamse altos coeficientes de mortalidade por AIDS em Porto Alegre, particularmente nos primeiros anos de estudo (1996-2001), chegando a 9 óbitos

Tabela 2. Variação anual percentual dos coeficientes de mortalidade por AIDS obtidas pela jointpoint regression, segundo faixa etária e região de residência, 1996 a 2012.

\begin{tabular}{lrrr}
\hline & Período & $\begin{array}{c}\text { APC } \\
(\%)\end{array}$ & IC(95\%) \\
\hline $\begin{array}{l}\text { Faixa Etária } \\
<1 \text { ano }\end{array}$ & $1996-2003$ & $-11,14$ & $-12,11$ a $-10,16$ \\
& $2003-2011$ & $-1,58$ & $-2,49$ a $-0,66$ \\
1 a 4 anos & $1996-1998$ & 4,94 & $-6,48$ a 16,36 \\
& $1998-2006$ & $-2,07$ & $-3,45$ a $-0,69$ \\
& $2006-2011$ & 0,01 & $-2,28$ a 2,29 \\
5 a 9 anos & $1996-2011$ & $-0,14$ & $-0,21$ a $-0,07$ \\
10 a 14 anos & $1996-2007$ & 0,47 & 0,25 a 0,70 \\
& $2007-2011$ & $-1,23$ & $-3,28$ a 0,83 \\
Região & & & \\
Sul & $1996-2001$ & $-0,01$ & $-0,04$ a 0,01 \\
& $2001-2004$ & $-0,20$ & $-2,43$ a 2,02 \\
Rio Grande & $2004-2012$ & $-0,03$ & $-0,05$ a $-0,01$ \\
do Sul & $1996-2012$ & $-0,13$ & $-0,14$ a $-0,12$ \\
Porto Alegre & $1996-1998$ & 0,10 & $-0,27$ a 0,46 \\
& $1998-2004$ & $-1,20$ & $-2,16$ a $-0,23$ \\
& $2004-2012$ & $-0,26$ & $-0,51$ a $-0,01$ \\
\hline
\end{tabular}

"APC: Annual Percentual Change (Variação Anual Percentual), $\mathrm{p}<0,05$. "*Rio Grande do Sul sem as ocorrências da capital. por 100 mil habitantes. Entretanto, registra-se queda acentuada no período seguinte, com tendência decrescente da curva suavizada, chegando a 1 óbito por 100 mil crianças da faixa etária (Figura 1B).

Quando se analisa a distribuição dos casos por faixas de idade, a incidência entre os menores de um ano supera todas as outras no período, apresentando em 1998, 2007 e 2011 picos de incidência maiores que a somatória de todas as outras faixas etárias. Observa-se elevação dos coeficientes de 2009 até 2011 (Figura 2A).

A redução da mortalidade entre crianças e adolescentes no período ocorreu de forma progressiva até 2011, porém os indicadores entre os menores de um ano se mantêm superiores aos de outras faixas etárias (Figura 2B). A tendência nesta faixa explica o formato da curva da Figura $1 \mathrm{~B}$, referente ao indicador de mortalidade de todas as crianças.

A regressão segmentada mais adequada para ajustar os coeficientes de mortalidade por AIDS de menores de 1 ano foi aquela com apenas um ponto de mudança no ano de 2003 , ou seja, foram suficientes dois segmentos de reta para representar a tendência decrescente dos coeficientes no período de 1996 a 2011 (Figura 3A). Já com relação aos coeficientes de Porto Alegre, o melhor ajuste da regressão segmentada foi com dois pontos de mudança, nos anos de 1998 e 2004. Assim, ajustou-se a tendência decrescente dos coeficientes de mortalidade com três segmentos de reta (Figura 3B).
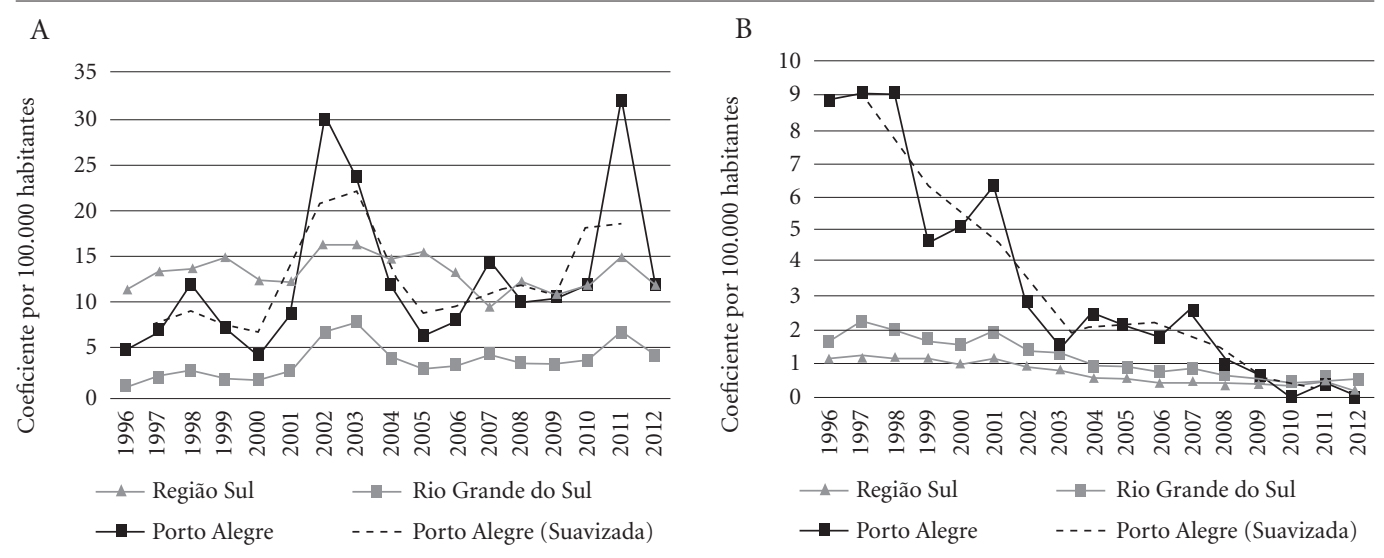

Figura 1. Coeficientes de Incidência (A) e Mortalidade (B) (por 100.00 habitantes) associados à AIDS (CID 10: B.20 a B.24) em indivíduos menores de 14 anos segundo ano de diagnóstico e região de estudo, Porto Alegre (com série suavizada por médias móveis de três pontos), Rio Grande do Sul e região Sul, 1996 a 2012. 
A

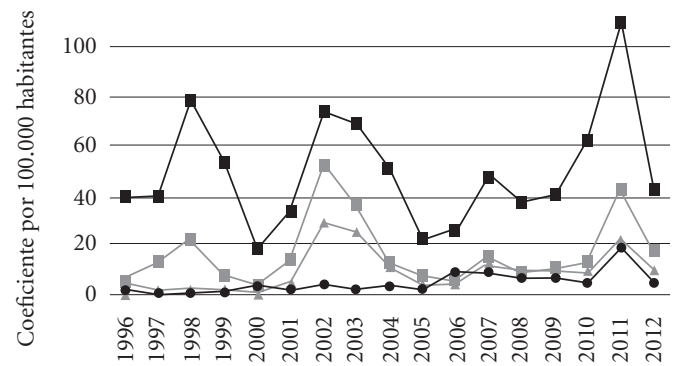

B

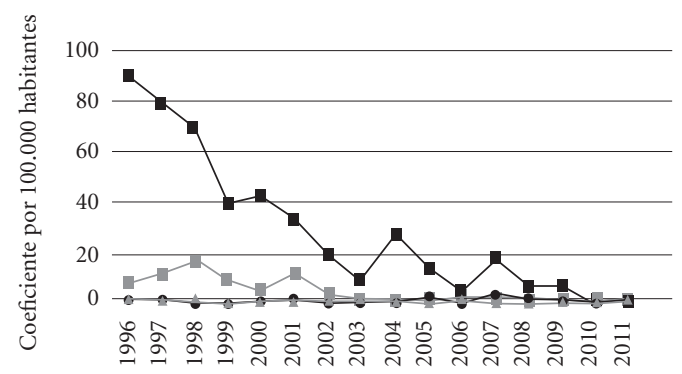

$\rightarrow-<1$ ano $\longrightarrow 1$ a 4 anos $\longrightarrow 5$ a 9 anos $\multimap 10$ a 14 anos

$-<1$ ano $\longrightarrow 1$ a 4 anos $\longrightarrow 5$ a 9 anos $\longrightarrow 10$ a 14 anos

Figura 2. Coeficientes de Incidência (A) e Mortalidade (B) (por 100.00 habitantes) associados à AIDS (CID 10: B.20 a B.24) segundo ano de diagnóstico e faixas etárias menores de 14 anos, Porto Alegre, 1996 a 2012.

A

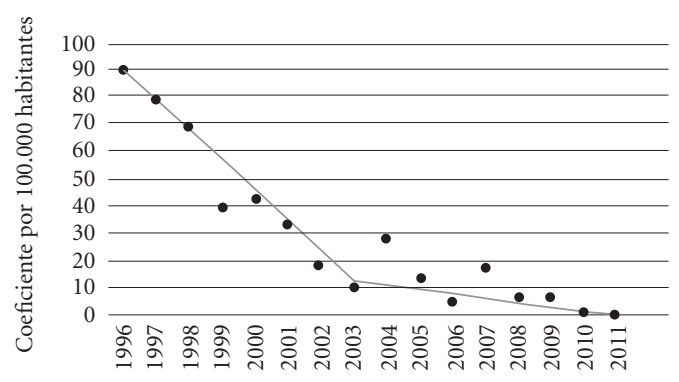

- $<1$ ano
B

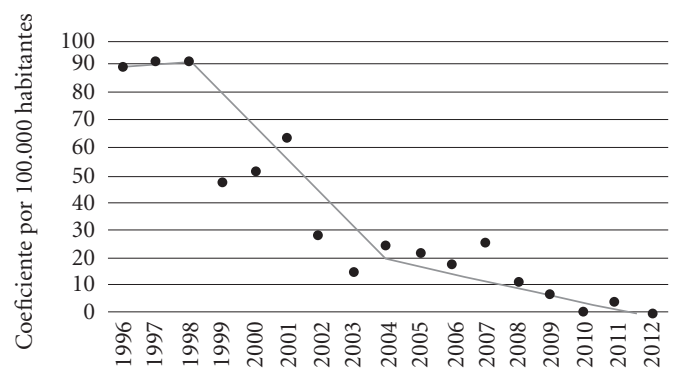

- 0-14 anos - 0-14 anos (ajustada)

Figura 3. Coeficientes de Mortalidade por 100.00 habitantes em menores de 1 ano (A) e de 0-14 anos (B) associados à AIDS (CID 10: B.20 a B.24) segundo ano de diagnóstico, obtidos com regressão segmentada (joinpoint regression), Porto Alegre, 1996 a 2012.

\section{Discussão}

Neste estudo observaram-se elevados coeficientes de incidência de AIDS em menores de 14 anos em Porto Alegre quando comparados aos do Estado e da região Sul do país. A faixa etária de menores de um ano foi a de maior notificação de casos, com pico no ano de 2007. O seguimento da criança exposta, a partir do nascimento, justifica a concentração do diagnóstico definitivo nos primeiros dois anos de vida ${ }^{17}$. Por outro lado, registra-se queda acentuada na mortalidade no período de estudo, principalmente entre os menores de um ano. $\mathrm{O}$ acesso aos protocolos de atendimento pediátrico, o seguimento clínico e o uso de antirretrovirais por parte das gestantes e dos recém-nascidos expostos ao HIV certamente tiveram impacto na sobrevida das crianças ${ }^{17}$.

O pico de incidência de AIDS em 2007 possivelmente está relacionado com a queda na Contagem da População ${ }^{12}$ de Porto Alegre do referido ano (22.421 hab. $<1$ ano em 2006 para 16.882 hab. $<1$ ano em 2007), ocasionando assim o aumento do coeficiente devido aos ajustes no denominador na faixa etária em questão, no município e no estado.

O perfil sociodemográfico (sexo e idade) dos indivíduos foi semelhante em Porto Alegre e no restante do estado ( $p>0,05)$, como também encontrado em estudos no Nordeste do país ${ }^{18,19}$. A 
diminuição do risco de AIDS em menores de 14 anos tem sido observada em várias regiões do Brasil após o acesso à terapia antirretroviral de alto impacto e a incorporação da sorologia no pré-natal e parto, como também relatado em outros países da América Latina ${ }^{20,21}$.

Os percentuais de casos e óbitos em indivíduos do estado e da capital que se referem como negros e pardos podem refletir a distinta distribuição racial na população de estudo. No Brasil, o percentual de casos de AIDS em indivíduos que se designam negros ou pardos foi de $54 \%$ em 2013 , superior ao encontrado em Porto Alegre 29,6\% ${ }^{11}$.

Alguns autores associam maior risco da infecção pelo HIV a fatores sociais e estruturais como: estigma, discriminação por gênero e raça ${ }^{22}$. Entre os determinantes sociais, a raça e as condições socioeconômicas são consideradas barreiras para o acesso à prevenção e à assistência à AIDS, embora poucos estudos tenham sido desenvolvidos no Brasil ${ }^{23}$. Há diferenças regionais com relação à cobertura do teste de HIV disponibilizado para as gestantes, associada a fatores como: raça, nível educacional e tamanho do município. Estas coberturas têm variado de $40,6 \%$ no Nordeste a $85,5 \%$ no Sul do Brasil ${ }^{24,25}$. O conceito de vulnerabilidade social e programática contribui para as análises das diferenças de raça, gênero e classe social, relacionando-as às dificuldades de acesso aos serviços e às informações referentes à doença ${ }^{26,27}$.

A redução da mortalidade por AIDS em crianças e adolescentes observada neste estudo tem sido reportada por outros autores no Brasil e em outros países, evidenciado a importância do acesso à HAART ao longo dos anos ${ }^{21,28-30}$. A mortalidade de menores de 5 anos caiu de 2 para 0,2 por 100 mil de 1996 a $2011^{31}$.

A mortalidade relacionada à AIDS em Porto Alegre tem sido nitidamente maior que em outras regiões do estado e país. Embora se encontrem poucos trabalhos sobre a mortalidade por AIDS em crianças no Brasil, o indicador de incidência publicado pelo Ministério da Saúde em menores de cinco anos é um marcador proxy utilizado para monitorar a transmissão vertical do HIV no país ${ }^{4}$.

Com menor coeficiente de incidência, inferior a 10/100.000 em 2012, o acometimento de crianças de 10 a 14 anos no estudo chegou a 15\% do total de casos. Vulnerabilidades do adolescente, como consumo de drogas, situações de rua e baixa escolaridade, têm sido apontadas como fatores associados à infecção HIV/AIDS ${ }^{32,33}$.

Embora se registre o impacto do Programa Nacional de DST e AIDS $^{4}$ na morbimortalidade por AIDS em várias regiões do país, ainda há a ocorrência de expressivo número de crianças infectadas. $\mathrm{O}$ acesso às medidas de prevenção e assistência aos pacientes é desigual segundo as condições sociodemográficas, como registram vários estudos ${ }^{18,34,35}$. Nem sempre os protocolos de assistência e prevenção são implementados com a mesma qualidade e garantia de acesso $^{36}$.

A incorporação do teste de HIV para a gestante na rotina nacional do pré-natal, e no Rio Grande do Sul, também durante o parto, reforçam os cuidados imediatos ao recém-nascido. $\mathrm{O}$ uso de antirretrovirais nas primeiras horas do nascimento (AZT ou AZT + nevirapina), além do seguimento clínico da criança exposta, entre outras medidas de prevenção primárias e secundárias, pode ter impacto na incidência e mortalidade por AIDS na infância ${ }^{37}$.

Atualmente, Porto Alegre dispõe de Programa de Atenção à AIDS, que disponibiliza, durante o parto, o teste de HIV em gestantes sem acompanhamento pré-natal. Esta estratégia mostrouse factível em maternidades do Rio de Janeiro e Porto Alegre, aumentando a sensibilidade do sistema e a captação de casos, nesta oportunidade, para o diagnóstico, diminuindo assim os riscos da infecção pelo vírus ${ }^{26}$.

A integralidade da atenção aos pacientes e suas famílias também tem se mostrado associada à maior sobrevida em crianças e adolescentes ${ }^{30}$. Tal realidade destaca a desigualdade regional das formas de diagnóstico e prevenção da infecção pelo HIV/AIDS, as quais ainda hoje são melhores nas regiões Sudeste e Sul do país. Por outro lado, os resultados deste estudo contradizem as altas coberturas do programa DST/AIDS e do acesso ao cuidado verificados na região Sul do país. A concentração das ações de prevenção e controle em hotspots, proposta pelo Ministério da Saúde 9 , pode lograr impacto na transmissão da AIDS nesta população de estudo.

Vale destacar que o plano integrado de ações UNAIDS/Porto Alegre para o enfrentamento da epidemia do HIV/AIDS no município, o AIDS Tchê, na forma como se organiza, possivelmente capta casos mais precocemente, aumentando a notificação, justificando as maiores taxas de detecção.

As duas abordagens de análise da tendência utilizadas neste estudo, a técnica de suavização por médias móveis e a regressão segmentada (joinpoint regression), foram complementares. Ambas têm sido amplamente utilizadas em estudos de análise de tendência (ou de séries temporais) com o intuito de identificar pontos sig- 
nificativos de mudança por meio da estimação da variação percentual média anual ${ }^{16}$. A primeira permitiu a melhor visualização do comportamento de tendências e de possíveis pontos de mudança e a segunda a identificação desses pontos, assim a como estimação dos incrementos em cada segmento de reta.

Algumas limitações podem ser apontadas neste estudo, entre elas a utilização de dados secundários e a possível subnotificação de casos, o que poder trazer distorções nas tendências, como também na construção de indicadores com números pequenos (numeradores), além de oscilações nas estimativas populacionais (denominadores). Estas podem interferir nas séries temporais, porém o aprimoramento dos registros e das estatísticas oficiais têm sido constantes, além da utilização crescente destas informações pelos serviços de saúde. Destaca-se a relevância dos indicadores construídos com banco de dados nacionais de incidência e mortalidade, ferramentas acessíveis para o planejamento de políticas públicas.

Uma limitação da regressão segmentada é que nem sempre os pontos de mudanças por ela identificados coincidem com os reais pontos e variações, uma vez que as regressões são conectadas continuamente ao longo do tempo ${ }^{14}$. Por outro lado, as especificidades culturais, socioeconômicas e políticas podem imprimir tendências diferentes às da epidemia na região Sul.

Destaca-se a capacidade do Programa HIV/ AIDS de Porto Alegre em captar novos casos e em notificá-los, aumentado os registros da epidemia. Por outro lado, o perfil da epidemia na região Sul, com as particularidades culturais e socioe- conômicas da população, pode esclarecer parte das diferenças no risco de adoecer e morrer na região. Neste sentido, as diretrizes de prevenção e assistência ao HIV/AIDS concebidas no âmbito federal não se configuram como as únicas possibilidades de ação no âmbito local. Ações pactuadas junto aos governos locais, sociedade civil e profissionais de saúde podem resultar em impacto positivo na prevenção e controle.

\section{Conclusão}

Os indicadores epidemiológicos de morbimortalidade da AIDS em Porto Alegre ainda superam os registrados no estado, na região Sul e em outras regiões do país.

Os coeficientes de mortalidade por AIDS apresentaram tendência decrescente no período de 1996 a 2012 para todas as faixas etárias (exceto de 10 a 14 anos) e regiões de estudo. Os declínios mais expressivos foram para a faixa etária de crianças menores que 1 ano, o que pode estar relacionado a ações de tratamento/prevenção às mães com uso de antirretrovirais durante a gestação, na capital Porto Alegre ${ }^{24,25}$.

Estudos de tendência de indicadores de risco e de mortalidade podem contribuir com os programas de controle de DST/AIDS por meio de diagnóstico epidemiológico que sugiram pontos vulneráveis, como: acesso aos serviços, qualidade da atenção, heterogeneidade do cuidado e grupos vulneráveis. Tais análises aprimoram a assistência da rede de cuidado para intervenções específicas e investimentos com maior potencial de impacto.

\section{Colaboradores}

MC Melo trabalhou na concepção, elaboração das análises, revisão do texto, discussão dos resultados e redação final. MR Donalísio trabalhou na concepção, elaboração das análises, revisão do texto, discussão dos resultados e redação final. JL do Nascimento trabalhou na revisão do texto e discussão dos resultados e redação final. RO Ferraz trabalhou na concepção, elaboração das análises, revisão do texto, discussão dos resultados e redação final. 


\section{Referências}

1. Polk BF, Fox R, Brookmeyer R, Kanchanaraksa S, Kaslow R, Visscher B, Rinaldo C, Phair J. Predictors of the acquired immunodeficiency syndrome developing in a cohort of seropositive homosexual men. $N$ Engl J Med 1987; 316(2):61-66.

2. Souza BB, Vasconcelos CC, Tenório DM, Alves Lucena MG, Holanda RLT. A Política de AIDS no Brasil: uma abordagem histórica. J Manag Prim Health Care 2010; 1(1):23-26.

3. Joint United Nation Programme on HIV/AIDS (UNAIDS). Global AIDS response progress reporting 2014: construction of core indicators for monitoring the 2011 United Nations political declaration on HIV/AIDS. Geneva: UNAIDS; 2014.

4. Brasil. Ministério da Saúde (MS). Secretaria de Vigilância em Saúde. Programa Nacional de DST e AIDS. Boletim Epidemiológico - AIDS e DST. Brasília: MS; 2011, 2012, 2013.

5. Brito AM, Sousa JL, Luna CF, Dourado I. Trends in maternal-infant transmission of AIDS after antiretroviral therapy in Brazil. Rev Saude Publica 2006; 40(Supl.):1822.

6. Foster G. Today's children-challenges to child health promotion in countries with severe AIDS epidemics. AIDS Care 1998; 10(Supl. 1):S17-S23.

7. Machado DM, Succi RC, Turato ER. Transitioning adolescents living with HIV/AIDS to adult-oriented health care: an emerging challenge. Jornal de Pediatria 2010; 86(6):465-472.

8. Gortmaker SL, Hughes M, Cervia J, Brady M, Johnson GM, Seage GR 3rd, Song LY, Dankner WM, Oleske JM; Pediatric AIDS Clinical Trials Group Protocol 219 Team. Effect of combination therapy including protease inhibitors on mortality among children and adolescents infected with HIV-1. N Engl J Med 2001; 345(21):1522-1528.

9. Brown LK, Lourie KJ, PaoM. Children and adolescents living with HIV and AIDS: a review. J Child Psychol Psychiatry 2000; 41(1):81-96.

10. Taquette SR, Matos HJ, Rodrigues AO, Bortolotti LR, Amorim E. A epidemia de AIDS em adolescentes de 13 a 19 anos, no município do Rio de Janeiro: descrição espaço-temporal. Rev Soc Bras Med Trop 2011; 44(4):467-470.

11. Brasil. Ministério da Saúde (MS). Secretaria de Vigilância em Saúde. Programa Nacional de DST e AIDS. Boletim Epidemiológico - AIDS e DST. Brasília: MS; 2014. Instituto Brasileiro de Geografia e Estatística (IBGE).

12. Censos demográficos: 2000 e 2010. Contagem Populacional: 1996. Estimativas preliminares para os anos intercensitários dos totais populacionais, estratificadas por idade e sexo pelo MS/SGEP/Datasus: 1992-1999, 2001-2006. Estimativas elaboradas no âmbito do Projeto UNFPA/IBGE (BRA/4/P31A) - População e Desenvolvimento. Coordenação de População e Indicadores Sociais: 2007-2009. Estimativas populacionais enviadas para o TCU, estratificadas por idade e sexo pelo MS/SGEP/Datasus: 2011-2012. Rio de Janeiro: IBGE; 2014.

13. Kim HJ, Fay MP, Feuer EJ, Midthune DN. Permatation tests for joinpoint regression with applications to cancer rates. Stat Med 2000; 19(3):335-351.
14. Ferreira DB, Mattos IE. Tendência da mortalidade por câncer de mama em mulheres no estado do Rio de Janeiro, Brasil, 1996-2011. Cien Saude Colet 2015; 20(3):895-903.

15. Joinpoint Regression Program, version 4.1.1.5 February 2015; Statistical Research and Applications Branch, National Cancer Institute.

16. Martinez-Beneito MA, Garcia-Donato G, Salmerón D. Bayesian joipoint regression model with unknown number of break-points. Ann Appl Stat 2011; 3(5):2150-2168

17. Brasil. Ministério da Saúde (MS). Secretaria de Vigilância em Saúde. Programa Nacional de DST e AIDS. Recomendações para Terapia Antirretroviral em Crianças e Adolescentes Infectados pelo HIV: manual de bolso/ Ministério da Saúde, Secretaria de Vigilância em Saúde, Programa Nacional de DST e AIDS. Brasília: MS; 2009.

18. Maluf e Silva MJ, Mendes WS, Gama MEA, Chein MBC, Veras DS. Perfil clínico-laboratorial de crianças vivendo com HIV/AIDS por transmissão vertical em uma cidade do Nordeste brasileiro. Rev. Soc. Bras. Med. Trop. 2010; 43(1):32-35.

19. Machado MMT, Galvão MTG, Lindsay AC, Cunha AJLA, Leite AJM, Leite RD, Kerr LRFS. Condições sociodemográficas de crianças de zero a dois anos filhas de mães com HIV/AIDS, Fortaleza, CE, Brasil. Rev Bras Saúde Matern Infant 2010; 10(3):377-382.

20. Bastos FI, Cáceres C, Galvão J, Veras MA, Castilho EA. AIDS in Latin America: assessing the current status of the epidemic and the ongoing response. Int J Epidemiol 2008; 37(4):729-737.

21. Ramos Júnior AN, Matida LH, Hearst N, Heukelbach J. AIDS in Brazilian Children: History, Surveillance, Antiretroviral Therapy, and Epidemiologic Transition, 1984-2008. AIDS Patient Care STDS 2011; 25(4):245255

22. Logie C, James L, Tharao W, Loutfy M. Associations Between HIV-Related Stigma, Racial Discrimination, Gender Discrimination, and Depression Among HIV-Positive African, Caribbean, and Black Women in Ontario, Canada. AIDS Patient Care and STDs 2013; 27(2):114-122.

23. Monteiro S, Villela WV, Knauth D. Discrimination, stigma, and AIDS: a review of academic literature produced in Brazil (2005-2010). Cad Saude Publica 2012; 28(1):170-176

24. Veloso VG, Bastos FI, Portela MC, Grinsztejn B, João EC, Pilotto JH, Araújo AB, Santos BR, Fonseca RC, Kreitchmann R, Derrico M, Friedman RK, Cunha CB, Morgado MG, Saines KN, Bryson YJ. HIV rapid testing as a key strategy for prevention of mother-tochild transmission in Brazil. Rev Saude Publica 2010; 44(5):803-811

25. Szwarcwald CL, Barbosa Júnior A, Souza-Júnior PR, Lemos KR, Frias PG, Luhm KR, Holcman MM, Esteves MA. HIV testing during pregnancy: use of secondary data to estimate 2006 test coverage and prevalence in Brazil. Braz J Infect Dis 2008; 12(3):167-172. 
26. Ayres JRCM. O conceito de vulnerabilidade e as práticas de saúde: novas perspectivas e desafios. In: Czeresnia D, Freitas CM, organizadores. Promoção da saúde: conceitos, reflexões, tendências. Rio de Janeiro: Fiocruz; 2003. p. 117-139.

27. Ayres JRCM, França Júnior I, Calazans GJ, Saletti Filho HC. Vulnerabilidade e prevenção em tempos de AIDS. In: Barbosa RM, Parker RG, organizadores. Sexualidades pelo avesso: direitos, identidade e poder. São Paulo: Editora 34; 1999. p. 49-72.

28. Matida LH, Ramos Júnior AN, Heukelbach J, Hearst N; Brazilian Study Group on Survival of Children with AIDS. Continuing improvement in survival for children with acquired immunodeficiency syndrome in Brazil. Pediatr Infect Dis J 2009; 28(10):920-922.

29. Matida LH, Ramos Júnior AN, Heukelbach J, Sañudo A, Succi RC, Marques HH, Della Negra M, Hearst N. Improving survival in children with AIDS in Brazil: results of the second national study, 1999-2002. Cad Saude Publica 2011; 27(Supl. 1):s93-s103.

30. Ramos Junior AN, Matida LH, Hearst N, Heukelbach J. Opportunistic illnesses in Brazilian children with AIDS: results from two national cohort studies, 1983 2007. AIDS Res Ther 2011; 8:23.

31. Rocha DFNC, Montes LKV. Boletim Epidemiológico do Estado de Goiás. Goiânia: Secretaria Estadual de Saúde; 2013.

32. Pereira AGL, Matos HJ, Escosteguy CC, Marques MVRE, Medronho RA. Sobrevida de pacientes com Síndrome da Imunodeficiência Adquirida em hospital geral no Rio de Janeiro, a partir de dados da vigilância epidemiológica. Cad Saúde Colet 2013; 21(2):160-167.

33. Bassols AMS, Boni R, Pechansky F. Alcohol, drugs, and risky sexual behavior are related to HIV infection in female adolescents. Rev Bras Psiquiatr 2010; 32(4):361368.

34. Candiani TMS, Pinto J, Cardoso CA, Carvalho IR, Dias AC, Carneiro M, Goulart EA. Impact of highly active antiretroviral therapy (HAART) on the incidence of opportunistic infections, hospitalizations and mortality among children and adolescents living with HIV/ AIDS in Belo Horizonte, Minas Gerais State, Brazil. Cad Saude Publica 2007; 23(Supl. 3):S414-S423.

35. Guibu IA, Barros MBA, Donalísio MR, Tayra A, Alves MCGP. Survival of AIDS patients in the Southeast and South of Brazil: analysis of the 1998-1999 cohort. Cad Saude Publica 2011; 27(Supl. 1):579-592.

36. Melchior R, Nemes MIB, Basso CR, Castanheira ERL, Alves MTSB, Buchalla CM, Donini AA; Equipe QualiAids. Avaliação da estrutura organizacional da assistência ambulatorial em HIV/AIDS no Brasil. Rev Saude Publica 2006; 40(1):143-151.

37. Brasil. Ministério da Saúde (MS). Secretaria de Vigilância em Saúde. Departamento de DST, AIDS e Hepatites Virais. Protocolo Clínico e Diretrizes Terapêuticas para Manejo da Infecção pelo HIV em Crianças e Adolescentes. Brasília: MS; 2014

Artigo apresentado em 10/04/2015

Aprovado em 03/09/2015

Versão final apresentada em 05/09/2015 